\title{
ALGEBRAIC MERIDIANS OF KNOT GROUPS
}

\author{
$\mathrm{BY}$
}

CHICHEN M. TSAU

\begin{abstract}
We propose the conjecture that every automorphism of a knot group preserves the meridian up to inverse and conjugation. We establish the conjecture for all composite knots, all torus knots, most cable knots, and at most one exception for hyperbolic knots; moreover we prove that the Property $\mathrm{P}$ Conjecture implies our conjecture. We also investigate hyperbolic knots in more detail, and give an example of figure-eight knot group and its automorphisms.
\end{abstract}

We shall study the automorphic images of the meridian of a knot, especially the relationship between the automorphic images of the meridian and the conjugates of the meridian. After looking at various kinds of knots, for example, the composite knots, the torus knots, the cable knots and many others, we propose the following conjecture.

CONJECTURE. Every automorphic image of the meridian of a knot is a conjugate of the meridian or its inverse.

We shall establish this conjecture for all composite knots and all torus knots; for the classes of cable knots, hyperbolic knots, and satellite knots, we also obtain partial results that limit the number of possible 'bad' automorphic images. These partial results can be sharpened by a recent result of Culler, Gordon, Luecke and Shalen (announced at a 3-manifold conference at the Mathematical Sciences Research Institute in Berkeley in January 1985). We will indicate how these results can be improved in a remark at the end of $\S 2$.

We shall also show that our conjecture would be a consequence of the 'Property $\mathrm{P}$ Conjecture', although it is not clear how one might prove that if a particular knot has Property $\mathrm{P}$, then the knot obeys our conjecture on automorphisms.

The author would like to thank the referee for his valuable comments and for pointing out errors in the proof of Theorem 2.3 of the original paper.

1. Preliminaries. In this paper, we work in the piecewise linear category. Let $M$ and $N$ be manifolds, and let $f: M \rightarrow N$ be a map; the induced homomorphism of $f$ on either $\pi_{1}(M) \rightarrow \pi_{1}(N)$ or $H_{1}(M) \rightarrow H_{1}(N)$ is denoted by $f_{*}$. In particular, if $M$ is a submanifold of $N$, then $i_{*}$ is the inclusion-induced homomorphism. A knot is a piecewise linear homeomorphic image of the circle $S^{1}$ in the 3 -sphere $S^{3}$. Homeomorphism of spaces, isomorphism of groups, homotopy of maps and homological equivalence are denoted by $\cong, \approx, \simeq, \sim$, respectively. Let $M$ be a

Received by the editors March 14, 1984 and, in revised form, May 13, 1985.

1980 Mathematics Subject Classification. Primary 57M05, 57M25.

Key words and phrases. Knot manifold, knot group, presentation, automorphism of knot group, torus knot, composite knot, cable knot, hyperbolic knot, surgery manifold. 
manifold. The interior and boundary of $M$ are denoted by $\operatorname{Int}(M)$ and $\operatorname{Bdy}(M)$ respectively. A regular neighborhood of a knot $K$ in $S^{3}$ is denoted $N(K)$, and the closure of the complement of $N(K)$ is denoted $C^{3}(K)$. Note that $S^{3}-N(K) \cong$ $S^{3}-K$, and both are homotopy equivalent to $C^{3}(K)$. When the choice of basepoint is not important, the groups $\pi_{1}\left(S^{3}-K\right), \pi_{1}\left(C^{3}(K)\right)$, and $\pi_{1}\left(S^{3}-N(K)\right)$ are viewed as identical. There are, up to isotopy, unique simple closed curves $\mu$ and $\lambda$ on $\operatorname{Bdy}\left(C^{3}(K)\right)$ such that $\mu$ bounds a disk in $N(K)$, and $\lambda \sim 0$ in $C^{3}(K)$. A choice of the pair $(\mu, \lambda)$, with any fixed orientation, is called a preferred meridian and longitude pair for $K, C^{3}(K)$ and $N(K)$. We shall call a simple closed curve $\xi$ on $\operatorname{Bdy}\left(C^{3}(K)\right)$ a $(p, q)$-curve if $\xi \simeq \mu^{p} \lambda^{q}$ in $C^{3}(K)$, where $(\mu, \lambda)$ is a preferred meridian-longitude pair for $C^{3}(K)$.

Let $K$ be a knot with meridian $\mu$, and $\phi$ an automorphism of $\pi_{1}\left(S^{3}-K\right)$. If $\phi(\mu)$ is a conjugate of $\mu^{ \pm 1}$, then $\phi$ is called standard; otherwise $\phi$ is called exotic, and $\phi(\mu)$ is called an exotic algebraic meridian. Let $K_{1}$ and $K_{2}$ be two knots that lie on either side of a 2 -sphere $S^{2}$ such that $K_{1} \cap S^{2}=K_{2} \cap S^{2}$ is an arc $\alpha$. Then the composition of $K_{1}$ and $K_{2}$, denoted $K_{1} \# K_{2}$, is $\left(K_{1} \cup K_{2}\right)-\operatorname{Int}(\alpha)$. A knot $K$ in $S^{3}$ is said to be a composite knot if it is the composition of two knots in $S^{3}$. Let $p, q$ be relatively prime positive integers, and let $V$ be a standardly embedded solid torus in $S^{3}$ with a preferred meridian and longitude pair. A $(p, q)$-torus-knot is the knot which lies on $\operatorname{Bdy}(V)$ and wraps around $V$ in the meridianal direction $p$ times and in the longitudinal direction $q$ times.

Let $V^{\prime}$ be a standardly embedded solid torus in $S^{3}$, and let $K^{\prime}$ be a simple closed curve in $V^{\prime}$ that is not contained in a 3-cell in $V^{\prime}$ and is not a core of $V^{\prime}$. Let $K_{1} \subset S^{3}$ be another knot, and let $V=N\left(K_{1}\right)$ be a tubular neighborhood of $K_{1}$ in $S^{3}$. If $h: V^{\prime} \rightarrow V$ is a homeomorphism and $K=h\left(K^{\prime}\right)$, then $K$ is called a satellite knot about the knot $K_{1}$. In particular, if $K^{\prime}$ is a $(p, q)$-torus-knot on $\operatorname{Bdy}\left(V^{\prime}\right)$, and if $h$ sends a longitude on $\operatorname{Bdy}\left(V^{\prime}\right)$ to a longitude on $\operatorname{Bdy}(V)$, then $K=h\left(K^{\prime}\right)$ is called a $(p, q)$-cable-knot about $K_{1}$, and is denoted $K=J\left(p, q, K_{1}\right)$.

Note that if $K=K_{1} \# K_{2}$, then the exterior $C^{3}(K)$ can be expressed as a union $C^{3}\left(K_{1}\right) \cup C^{3}\left(K_{2}\right)$ along an annulus. If $K=J(p, q ; L)$, then $C^{3}(K)$ is a union $C^{3}(L) \cup T^{3}$ of $C^{3}(L)$ and a solid torus $T^{3}$ along an annulus.

2. Automorphisms of knot groups. We first consider the composite knot groups. A detailed proof of the following theorem can be found in the proof of Theorem 1 of [2]. For completeness of this paper, we shall include an outline of the proof.

\section{THEOREM 2.1. Every automorphism of a composite knot group is standard.}

PROOF. Let $K$ be a composite knot with meridian $\mu$, and let $\phi$ be an automorphism of $\pi_{1}\left(S^{3}-K\right)$. Since $S^{3}-K$ is aspherical, there is a homotopy equivalence $f: S^{3}-K \rightarrow S^{3}-K$ such that $f_{*}=\phi$.

Since $K$ is a composite knot, there exists a properly embedded essential annulus $A$ in $C^{3}(K)$ with $\operatorname{Bdy}(A)$ meridians of $K$. By [14, Lemma 1.1, p. 506], we may homotope $f$ so that $f$ is transverse with respect to $A$ and $f^{-1}(A)$ is a collection of properly embedded incompressible 2 -sided compact surfaces in $C^{3}(K)$. Choose such an $f$ with $f^{-1}(A)$ having minimum number of components. Since $f_{*}=\phi$, which is injective, $f^{-1}(A)$ contains only disks or annuli; but since $\operatorname{Bdy}(M)$ is incompressible in $M$ and $M$ is aspherical, $f^{-1}(A)$ contains no disks. 
Let $A^{\prime}$ be an annulus in $f^{-1}(A)$. Then $\left.f\right|_{A^{\prime}}$ is a homotopy equivalence, hence there exists a homeomorphism $h: A^{\prime} \rightarrow A$ with $\left.f\right|_{A^{\prime}}=h$. Since $f$ is transverse to $A$, we may extend the homotopy of $\left.f\right|_{A^{\prime}}$ to $C^{3}(K)$, so we may assume $\left.f\right|_{A^{\prime}}$ is a homeomorphism for each component $A^{\prime}$ in $f^{-1}(A)$.

By $[\mathbf{1 1}]$, we may also assume that $f^{-1}(A)$ has only one component $A^{\prime}$. By Lemma 1 and Lemma 2 of $[\mathbf{2}]$, and also the fact that cable knots are prime [9], we may conclude that each boundary component of $A^{\prime}$ is a meridian of $K$. Since $\left.f\right|_{A^{\prime}}: A^{\prime} \rightarrow A$ is a homeomorphism, $f$ preserves the meridian of $K$. This completes the proof.

THEOREM 2.2. Every automorphism of a torus knot group is standard.

ProOF. Let $K$ be a $(p, q$,$) -torus-knot (p, q \in Z$, and $(p, q)=1)$. Let $G=$ $\pi_{1}\left(S^{3}-K\right)$. Then $G$ has the presentation of the form $G=\left\langle a, b: a^{p}=b^{q}\right\rangle$. By $[\mathbf{1 0}]$, the automorphism group of $G$ has the presentation

$$
\operatorname{Aut}(G)=\left\langle I, J, H: I^{p}=J^{q}=H^{2}=(H I)^{2}=(H J)^{2}=1\right\rangle,
$$

where $I$ is conjugation by $a, J$ is conjugation by $b$, and $H(a)=a^{-1}, H(b)=b^{-1}$. Thus it is sufficient to prove that each generator of the automorphism group $\operatorname{Aut}(G)$ sends a meridian to a conjugate of it or its inverse. This is obvious for generators $I$ and $J$. In $G$ the meridian $\mu$ of $K$ can be represented as $\mu=b^{r} a^{s}$, where $r, s \in Z$ and satisfy $p r+q s=1$. Then $H(\mu)=b^{-r} a^{-s}=a^{s}\left(a^{-s} b^{-r}\right) a^{-s}=a^{s-1} a^{-s}$ is a conjugate of $\mu^{-1}$. The proof is completed.

THEOREM 2.3. Every nontrivial cable knot admits at most one exotic algebraic meridian (up to conjugation and inverse).

ProOF. Let $K=J(p, q ; L)$ be a $(p, q)$-cable-knot about a knot $L ; p, q \in Z$ and $(p, q)=1$. Represent $C^{3}(K)$ as the union of $C^{3}(L)$ and a solid torus $V$ along an annulus $A$. Let $(\mu, \lambda)$ be a meridian-longitude pair for $K$, and let $\left(\mu_{L}, \lambda_{L}\right)$ be a meridian-longitude pair for $L$. Since each component of $A$, when properly oriented, is a $(p, q)$-curve on $\operatorname{Bdy}\left(C^{3}(L)\right)$, by Van Kampen's theorem, we can represent $\pi_{1}\left(C^{3}(K)\right)$ as the amalgamated free product $\pi_{1}\left(C^{3}(K)\right)=\pi_{1}\left(C^{3}(L)\right) *_{\mu^{p} \lambda^{q}=t^{q}}\langle t\rangle$, where $t$ is a generator of $\pi_{1}(V) \approx Z$.

Let $\phi$ be an automorphism of $\pi_{1}\left(C^{3}(K)\right)$. By the proof of Theorem 2 in [2], there is a homotopy equivalence $h: C^{3}(K) \rightarrow C^{3}(K)$ such that $h_{*}=\phi$, and there is a knot $L^{\prime}$ such that $K$ is a cable knot about $L^{\prime}$ and $h \mid C^{3}\left(L^{\prime}\right): C^{3}\left(L^{\prime}\right) \rightarrow C^{3}(L)$ is a homeomorphism. We can represent $C^{3}(K)$ as the union of $C^{3}\left(L^{\prime}\right)$ amid a solid torus $V^{\prime}$ along an annulus $A^{\prime}$, and we have $h\left(A^{\prime}\right)=A,\left.h\right|_{V^{\prime}}: V^{\prime} \rightarrow V$ is a homotopy equivalence. By $[\mathbf{9}], K=J\left(p, q ; L^{\prime}\right)$, and $L^{\prime}$ is of the same isotopy type of $L$.

Choose a point $x \in A$ as basepoint, and let $x^{\prime} \in A^{\prime}$ be such that $h\left(x^{\prime}\right)=x$. Let $\left(\mu_{L^{\prime}}, \lambda_{L^{\prime}}\right)$ be the meridian and longitude pair for $L^{\prime}$. Since $h: C^{3}\left(L^{\prime}\right) \rightarrow C^{3}(L)$ is a homeomorphism, we may assume that $h\left(\mu_{L^{\prime}}\right)=\mu_{L}^{a} \lambda_{L}^{m}$ and $h\left(\lambda_{L^{\prime}}\right)=\lambda_{L}^{b}$ for some $a, b$ and $m \in Z$ and $|a|=|b|=1$. Each boundary of $A^{\prime}$, when properly oriented, is a $(p, q)$-curve on $\operatorname{Bdy}\left(C^{3}\left(L^{\prime}\right)\right)$, so $h\left(\mu_{L^{\prime}}^{p} \lambda_{L^{\prime}}^{q}\right)=\left(\mu_{L}^{p} \lambda_{L}^{q}\right)^{c}$, where $c=1$ or -1. Recomputing $h\left(\mu_{L^{\prime}}^{p} \lambda_{L^{\prime}}^{q}\right)$ in terms of $h\left(\mu_{L^{\prime}}\right)$ and $h\left(\lambda_{L^{\prime}}\right)$, we obtain

$$
h\left(\mu_{L^{\prime}}^{p} \lambda_{L^{\prime}}^{q}\right)=\left(\mu_{L}^{a} \lambda_{L}^{m}\right)^{p}\left(\lambda_{L}^{b}\right)^{q}=\mu_{L}^{a p} \lambda_{L}^{m p+b q} .
$$

Therefore we have $\left(\mu_{L}^{p} \lambda_{L}^{q}\right)^{c}=\mu_{L}^{a p} \lambda_{L}^{m p+b q}$; hence $a p=p c$, so $a=c$, and $m p+b q=$ $q c=q a$, so $m p=q(a-b)$. 
Consider the following two cases.

Case (1). $m=0$. Then $a=b$ and so $h\left(\mu_{L^{\prime}}\right)=\mu_{L}^{a}$ and $h\left(\lambda_{L^{\prime}}\right)=\lambda_{L}^{a}$.

Case (2). $m \neq 0$. Then $b=-a$ and so $m=2 a q / p$. This implies that $p$ is an integral factor of 2 , and $h\left(\mu_{L^{\prime}}\right)=\mu_{L}^{a} \lambda_{L}^{2 a q / p}, h\left(\lambda_{L^{\prime}}\right)=\lambda_{L}^{-a}$.

We can also represent $\pi_{1}\left(C^{3}(K)\right)$ as the amalgamated free product

$$
\pi_{1}\left(C^{3}(K)\right)=\pi_{1}\left(C^{3}(L)\right) *_{\mu_{L}^{p}, \lambda_{L \prime}^{q}=t^{\prime q}}\left\langle t^{\prime}\right\rangle,
$$

where $t^{\prime}$ is a generator for $\pi_{1}\left(V^{\prime}\right) \approx Z$. Now because of the relations $\mu_{L}^{p} \lambda_{L}^{q}=t^{q}$ and $\mu_{L^{\prime}}^{p} \lambda_{L^{\prime}}^{q}=t^{\prime q}$, and because (recall $\left.c=a\right) h\left(\mu_{L^{\prime}}^{p} \lambda_{L^{\prime}}^{q}\right)=\left(\mu_{L}^{p} \lambda_{L}^{q}\right)^{a}$, we have $h\left(t^{\prime}\right)=t^{a}$.

The meridian $\mu$ of $K$ can be presented as $\mu=\mu_{L^{\prime}}^{r} \lambda_{L^{\prime}}^{s} t^{\prime-s}$ when considered as in $\pi_{1}\left(C^{3}\left(L^{\prime}\right)\right) * \mu_{L^{\prime}}^{p} \lambda_{L^{\prime}}^{q}=t^{\prime q}\left\langle t^{\prime}\right\rangle$, or as $\mu_{L}^{r} \lambda_{L}^{s} t^{-s}$ when considered as in $\pi_{1}\left(C^{3}(L)\right) * \mu_{L}^{p} \lambda_{L}^{q}=t^{q}$ $\langle t\rangle$, where $r, s \in Z$ satisfy $q r-p s=1$.

Therefore in case (1), for $a=1$ we have

$$
\phi(\mu)=\phi\left(\mu_{L^{\prime}}^{r} \lambda_{L^{\prime}}^{s} t^{\prime-s}\right)=\mu_{L}^{r} \lambda_{L}^{s} t^{-s}=\mu ;
$$

and for $a=-1$ we have

$$
\phi(\mu)=\mu_{L}^{-r} \lambda_{L}^{-s} t^{s}=t^{-s}\left(t^{s} \mu_{L}^{-r} \lambda_{L}^{-s}\right) t^{s}=t^{-s} \mu^{-1} t^{s} .
$$

Hence $\phi(\mu)$ is a conjugate of $\mu^{ \pm 1}$.

In case (2), for $a=1$ we have

$$
\begin{aligned}
\phi(\mu) & =\phi\left(\mu_{L^{\prime}}^{r} \lambda_{L^{\prime}}^{s} t^{\prime-s}\right)=\left(\mu_{L} \lambda_{L}^{2 q / p}\right)^{r} \lambda_{L}^{-s_{t}-s} \\
& =\mu_{L}^{r} \lambda_{L}^{(2 q r / p)-s} t^{-s}=\mu_{L}^{r} \lambda_{L}^{(q r+1) / p^{-s}} t^{-s}
\end{aligned}
$$

and for $a=-1$ we have

$$
\phi(\mu)=\mu_{L}^{-r} \lambda_{L}^{-2 q r / p+s} t^{s}=\mu_{L}^{-r} \lambda_{L}^{-(q r+1) / p} t^{s}=t^{-s}\left(t^{s} \mu_{L}^{-r} \lambda_{L}^{-(q r+1) / p}\right) t^{s} .
$$

Let $w=\mu_{L}^{r} \lambda_{L}^{(q r+1) / p} t^{-s}$. We have in this case that $\phi(\mu)$ is a conjugate of $w^{ \pm 1}$ for fixed $w \in \pi_{1}\left(C^{3}(K)\right)$. The proof is completed.

From the proof of Theorem 2.3, we have

COROllary 2.4. Only those $(p, q)$-cable-knots with $|p|=1$ or 2 can possibly admit exotic algebraic meridians.

Let $K$ be a knot in $S^{3}$. The $(p, q)$-surgery-manifold, denoted $(K ; p / q)$, is formed by identifying the boundary of a solid torus to the boundary of $C^{3}(K)$ so that a $(p, q)$-curve on $\operatorname{Bdy}\left(C^{3}(K)\right)$ is identified with a meridian curve of the solid torus. A knot $K$ in $S^{3}$ is said to have Property $\mathrm{P}$ if $(K ; p / q)$ is non-simply-connected for all $(p, q)=1$ except $(K ; \pm 1 / 0) \cong S^{3}$.

THEOREM 2.5. Modulo the Property P Conjecture, every automorphism of a knot group is standard.

ProOF. Let $K$ be a knot in $S^{3}$, and let $\phi$ be an automorphism of $\pi_{1}\left(C^{3}(K)\right)$. We consider the following two cases: (1) The knot manifold $C^{3}(K)$ contains a properly embedded essential annulus. Then by $[\mathbf{2}], K$ is either a composite knot, a torus knot, or a cable knot. By Theorems 2.1 and 2.2, composite and torus knots have only standard automorphisms. If $K$ is cable, then since case (2) of the proof of Theorem 2.3 implies that $L$ fails Property P, $\phi$ must be in case (1) and hence is standard. 
(2) The knot manifold contains no properly embedded essential annulus. Then by $[\mathbf{3}]$ and by the asphericity of knot manifolds, there is a homeomorphism $h: C^{3}(K) \rightarrow$ $C^{3}(K)$ such that $H_{*}=\phi$ (up to conjugation). Since $K$ has Property $\mathrm{P}$ by assumption, then $h(\mu)=\mu^{ \pm 1}$; hence $\phi$ is standard. The proof is completed.

REMARK. Note that Theorem 2.5 assumes Property $\mathrm{P}$ for all knots, it is a little bit too strong; actually, we observe from the proof of Theorem 2.5 that except in the case of cable knot, for any given knot $K$, we only need assume that the knot $K$ has Property P. It then follows that every automorphism of the $K$-knotgroup is standard. If $K$ is a cable knot about a knot $L$, and $L$ has Property $\mathrm{P}$, then every automorphism of the $K$-knot-group is standard. Actually, Property $\mathrm{P}$ is also too strong in as much as it is only necessary to assume that $(K ; 1 / n)$ is not homeomorphic to $S^{3}$ for $n \neq 0$ (as opposed to assuming that it is not simply connected).

We now begin our discussion of hyperbolic knots. We shall show that certain hyperbolic knots obey our conjecture and, in any case, admit at most one possible exotic algebraic meridian.

A 3-manifold with $\operatorname{Bdy}(M)=\Phi$ is hyperbolic if $M$ admits a complete Riemannian metric of constant sectional curvature -1 with finite volume. If $M$ is a hyperbolic 3-manifold, then the universal covering space of $M$ can be taken as the hyperbolic upper half 3 -space, and $M$ is the orbit space of the hyperbolic 3 space modulo a discrete subgroup of the group of orientation preserving isometries. A knot $K$ is hyperbolic if the interior of the $K$-knot-manifold is a hyperbolic 3 manifold. By Thurston's uniformization theorem [12], a knot is hyperbolic if and only if it is neither a torus knot nor a satellite knot.

Let $K$ be a hyperbolic knot. Then by Thurston's finiteness theorem $[\mathbf{1 2}]$, the surgery manifolds $(K ; 1 / n)$ are hyperbolic manifolds for all but at most finitely many $n \in Z$. Let $m=\min \{n \mid(K ; 1 / n)$ is not hyperbolic $\}$, and let $M=$ $\max \{n \mid(K ; 1 / n)$ is not hyperbolic $\}$. Since $(K ; 1 / 0) \cong S^{3}$ is not hyperbolic, it is obvious that $m \leq 0 \leq M$.

THEOREM 2.6 (RILEY [8]). Each hyperbolic knot admits at most one exotic algebraic meridian (up to conjugation and inverse). Specifically, the only possible exotic algebraic meridian must be of the form $\mu^{a} \lambda^{c}$ where $a=1$ or -1 , and $c=$ $a(M+m)$.

PROOF. By Thurston's uniformization theorem [12], each automorphism of the fundamental group of a hyperbolic 3-manifold preserves the peripheral subgroup, therefore by $[\mathbf{1 3}]$, each automorphism is induced by a homeomorphism of the hyperbolic manifold. So if $K$ is a hyperbolic knot and $\phi$ is an automorphism of $\pi_{1}\left(C^{3}(K)\right)$, then there is a homeomorphism $h: C^{3}(K) \rightarrow C^{3}(K)$ such that $h_{*}=\phi$ (up to conjugation). Therefore it is sufficient to show either $h(\mu)=\mu^{a}$ or $h(\mu)=\mu^{a} \lambda^{c}$. By homology, $h(\mu)=\mu^{a} \lambda^{q}$ for some $q \in Z$. We show that if $q \neq 0$, then $q=c=a(M+m)$.

So assume $q \neq 0$. Let us first assume $a=1$, so $h(\mu)=\mu \lambda^{q}$. By homology, $h(\lambda)=\lambda^{ \pm 1}$. If $h(\lambda)=\lambda$, then $h^{2}(\mu)=h\left(\mu \lambda^{q}\right)=\left(\mu \lambda^{q}\right) \lambda^{q}=\mu \lambda^{2 q}$; by induction, we can show $h^{n}(\mu)=\mu \lambda^{n q}$ for any $n \in Z$. Since $h^{n}: C^{3}(K) \rightarrow C^{3}(K)$ is a homeomorphism, we have $(K ; 1 / n q) \cong(K ; 1 / 0) \cong S^{3}$; therefore $(K ; 1 / n q)$ are not hyperbolic for all $n \in Z$, which contradicts the finiteness theorem. Thus we must have $h(\lambda)=\lambda^{-1}$. 
Now $h\left(\mu \lambda^{m}\right)=\left(\mu \lambda^{q}\right) \lambda^{-m}=\mu \lambda^{q-m}$, and $h\left(\mu \lambda^{M}\right)=\mu \lambda^{q-M}$. So $(K ; 1 / q-m) \cong$ $(K ; 1 / m)$ and $(K ; 1 / q-M) \cong(K ; 1 / M)$. Since $(K ; 1 / m)$ and $(K ; 1 / M)$ are not hyperbolic, $(K ; 1 / q-m)$ and $(K ; 1 / q-M)$ are not hyperbolic, hence $m \leq q-M \leq$ $q-m \leq M$. But then we have $M+m \leq q$ and $q \leq M+m$, so $q=M+m$.

A similar proof applies to show that if $a=-1$, then $h(\mu)=\mu^{-1} \lambda^{q}, h(\lambda)=\lambda$ and $q=-(M+m)$.

From the proof of Theorem 2.6, we have

COROLLARY 2.7. Every exotic automorphism of a hyperbolic knot group is induced by an orientation reversing homeomorphism.

COROLlARY 2.8. Every automorphism of an amphicheiral hyperbolic knot group is standard.

PrOOF. Let $K$ be an amphicheiral hyperbolic knot. Then there is a homeomorphism $h: C^{3}(K) \rightarrow C^{3}(K)$ such that $h(\mu)=\mu$, and $h(\lambda)=\lambda^{-1}$. Therefore $h\left(\mu \lambda^{m}\right)=\mu \lambda^{-m}$, and $h\left(\mu \lambda^{M}\right)=\mu \lambda^{-M} ;$ hence we have $(K ; 1 /-m) \cong(K ; 1 / m)$ and $(K ; 1 /-M) \cong(K ; 1 / M)$. Thus the surgery manifolds $(K ; 1 /-m)$ and $(K ; 1 /-M)$ are nonhyperbolic, so $-m \leq M$ and $m \leq-M$; hence we have $M+m \geq 0$ and $M+m \leq 0$, therefore $M+m=0$. By Theorem 2.6, every automorphism of the $K$-knot-group must be standard.

REMARK. Theorem 2.6 and Corollaries 2.7 and 2.8, which are proved using Thurston's finiteness theorem, hold in greater generality, as noted by the referee as follows. Let $K$ be a knot that is neither composite, cabled, nor torus (so the $K$-knotmanifold $C^{3}(K)$ contains no properly embedded essential annulus). Then every automorphism $\phi$ of $\pi_{1}\left(C^{3}(K)\right)$ is induced by an autohomeomorphism of $C^{3}(K)$, and so $\phi(\mu)=\mu^{a} \lambda^{c}, \phi(\lambda)=\lambda^{\eta}(a= \pm 1, \eta= \pm 1, c \in Z)$. Hence $(K ; a / c) \cong S^{3}$. By the fact that torus knots have Property P, Thurston's finiteness theorem for hyperbolic Dehn surgery, and a finiteness theorem for satellite knots [4], we may conclude that $\left\{n \mid(J ; 1 / n) \cong S^{3}\right\}$ is finite for all $J$. Now using the same argument as in the proof of Theorem 2.6, we can prove that any $\phi$ with $a=\eta$ must have $c=0$, and hence $\phi$ is standard. Moreover, if we define $m=\min \left\{n \mid(J ; 1 / n) \cong S^{3}\right\}$ and $M=\left\{n \mid(J ; 1 / n) \cong S^{3}\right\}$, then if $\phi$ is an automorphism with $a=-\eta$, we can prove, again by the same argument as in the proof of Theorem 2.6, that $c=a(M+m)$. Therefore Theorem 2.6 and Corollary 2.7 can be extended to include all knots which are not composite, cabled, or torus.

Corollary 2.8 also holds for all amphicheiral knots which are not composite, cabled, or torus, as follows. If $K$ is amphicheiral of the above type, then there is an automorphism $h$ of $\pi_{1}\left(C^{3}(K)\right)$ such that $h(\mu)=\mu^{\epsilon}, h(\lambda)=\lambda^{-\epsilon}(\epsilon= \pm 1)$. Now if $\phi$ is any automorphism of $\pi_{1}\left(C^{3}(K)\right)$, from the above discussion we know that either $\phi(\mu)=\mu^{a}$ and $\phi(\lambda)=\lambda^{a}$ (so $\phi$ is standard), or $\phi(\mu)=\mu^{a} \lambda^{c}$ and $\phi(\lambda)=\lambda^{-a}$. In the second case we have $\phi h(\mu)=\mu^{a} \lambda^{c}$ and $h(\lambda)=\lambda^{a}$. This in turn implies $c=0$, and hence $\phi$ is standard. Actually, since cable knots and torus knots are not amphicheiral, it follows from above and Theorem 2.1 that Corollary 2.8 holds for all amphicheiral knots.

Because the interior of a hyperbolic knot manifold has extra geometric structure, we shall discuss hyperbolic knots in more detail.

Let $K$ be a hyperbolic knot and let $M=\operatorname{Int}\left(C^{3}(K)\right)$. Then $M$ has a hyperbolic structure, and the universal covering space of $M$ can be viewed as the hyperbolic 
upper half 3 -space $H^{3}=\{(z, t) \mid z \in C, t>0\}$. The fundamental group $\pi_{1}(M)$ is lifted to a discrete subgroup of the group of orientation preserving isometries of $H^{3}$ such that the orbit space $H^{3} / \pi_{1}(M)$ is $M$.

It is well known that the group of orientation preserving isometries of $H^{3}$ can be identified as $\operatorname{PSL}(2, C)=\operatorname{SL}(2, C) /\langle I,-I\rangle$, where $\operatorname{SL}(2, C)$ is the group of all 2 -by-2 complex matrices with determinant 1 . The group of isometries of $H^{3}$ (orientation preserving and orientation reversing isometries), denoted $\mathrm{P} \Gamma \mathrm{L}(2, C)$, is then identified as the $Z_{2}$-extension of $\operatorname{PSL}(2, C)$, obtained by adding an element $A^{*}$ to $\operatorname{PSL}(2, C)$ with the relations $\left(A^{*}\right)^{2}=1$ and $A^{*} g A^{*}=\bar{g}$ for any $g \in \operatorname{PSL}(2, C)$. Specifically, $A^{*}$ may be chosen so that the action of $\operatorname{P\Gamma L}(2, C)$ on $H^{3}$ can be extended to an action on $C^{*}=C \cup\{\infty\}$ (sphere at infinity for $H^{3}$ ) as follows: For $g=\left(\begin{array}{ll}a & b \\ c & d\end{array}\right) \in \operatorname{PSL}(2, C), g(z)=(a z+b) /(c z+d)$ is the Mobius transformation, and $A^{*}(z)=\bar{z}$.

Since $\left(A^{*}\right)^{2}=1$ and $A^{*} g A^{*}=\bar{g}$, we can write $\operatorname{P\Gamma L}(2, C)$ as $\operatorname{P\Gamma L}(2, C)=$ $\operatorname{PSL}(2, C) \cup\left\{A^{*} g \mid g \in \operatorname{PSL}(2, C)\right\}$. In particular, each orientation reversing isometry of $H^{3}$ is of the form $A^{*} g$ for some $g \in \operatorname{PSL}(2, C)$.

Let $\mu$ and $\lambda$ be the meridian and longitude of $K$. The subgroup $P$ generated by $\mu$ and $\lambda$ is isomorphic to $Z+Z$ and is conjugate in $\operatorname{PSL}(2, C)$ to

$$
\left\langle\left(\begin{array}{ll}
1 & 1 \\
0 & 1
\end{array}\right),\left(\begin{array}{ll}
1 & x \\
0 & 1
\end{array}\right)\right\rangle \text { for some } x \in C-R .
$$

For the remainder of this paper, we assume that any faithful representation of a hyperbolic knot group into $\operatorname{PSL}(2, C)$ has been normalized so that the meridian and longitude can be represented as

$$
\mu=\left(\begin{array}{ll}
1 & 1 \\
0 & 1
\end{array}\right) \quad \text { and } \quad \lambda=\left(\begin{array}{ll}
1 & x \\
0 & 1
\end{array}\right)
$$

According to Marden's version (Theorem 8.3 of [6]) of Mostow's rigidity theorem, we can identify the automorphism group $\operatorname{Aut}\left(\pi_{1}(M)\right)$ of $\pi_{1}(M)$ with the normalizer $\Omega$ of $\pi_{1}(M)$ in $\operatorname{PCL}(2, C)$, so that each automorphism $\phi$ is induced by an inner automorphism of $\mathrm{P} \Gamma \mathrm{L}(2, C)$. More precisely, the automorphism $\phi$ corresponds to a unique $T \in \Omega$ such that $\phi(U)=T U T^{-1}$ for every $U \in \pi_{1}(M) \subset \operatorname{PSL}(2, C)$.

Let $\phi \in \operatorname{Aut}\left(\pi_{1}(M)\right)$. Since $\phi$ is induced by a homeomorphism of $M, \phi(P)$ is conjugate to $P$. Hence up to conjugation we have $\phi(\mu)=\mu^{r} \lambda^{q}$ and $\phi(\lambda)=\lambda^{s}$, where $r, s, q \in Z$ and $|r|=|s|=1$.

We first consider the case where $\phi$ is induced by an orientation preserving homeomorphism of $M$. Then $r=s$ and by the above three paragraphs there exists $g \in \operatorname{PSL}(2, C)$ such that $g \mu g^{-1}=\phi(\mu)=\mu^{r} \lambda^{q}$ and $g \lambda g^{-1}=\phi(\lambda)=\lambda^{r}$.

Let $g=\left(\begin{array}{ll}a & b \\ c & d\end{array}\right)$. Then since $\mu=\left(\begin{array}{ll}1 & 1 \\ 0 & 1\end{array}\right)$ and $\lambda=\left(\begin{array}{ll}1 & x \\ 0 & 1\end{array}\right)$, we obtain the following two equations of matrices:

$$
\begin{aligned}
& \left(\begin{array}{ll}
a & b \\
c & d
\end{array}\right)\left(\begin{array}{ll}
1 & 1 \\
0 & 1
\end{array}\right)\left(\begin{array}{ll}
a & b \\
c & d
\end{array}\right)^{-1}=\left(\begin{array}{ll}
1 & 1 \\
0 & 1
\end{array}\right)^{r}\left(\begin{array}{ll}
1 & x \\
0 & 1
\end{array}\right)^{q} \\
& \left(\begin{array}{ll}
a & b \\
c & d
\end{array}\right)\left(\begin{array}{ll}
1 & x \\
0 & 1
\end{array}\right)\left(\begin{array}{ll}
a & b \\
c & d
\end{array}\right)^{-1}=\left(\begin{array}{ll}
1 & x \\
0 & 1
\end{array}\right)^{r}
\end{aligned}
$$


Multiplying, we obtain

$$
\begin{aligned}
& \left(\begin{array}{cc}
1-a c & a^{2} \\
-c^{2} & 1+a c
\end{array}\right)=\left(\begin{array}{cc}
1 & r+q x \\
0 & 1
\end{array}\right), \\
& \left(\begin{array}{cc}
1-a c x & a^{2} x \\
-c^{2} x & 1+a c x
\end{array}\right)=\left(\begin{array}{cc}
1 & r x \\
0 & 1
\end{array}\right) .
\end{aligned}
$$

From (1), we obtain $-c^{2}=0$, hence $c=0$; and $a^{2}=r+q x$. From (2), we obtain $a^{2} x=r x$. Since $x \neq 0$, thus $a^{2}=r$. Substituting $a^{2}=r$ in $a^{2}=r+q x$, we have $r=r+q x$, so $q x=0$; this implies $q=0$. Therefore $\phi(\mu)=\mu^{r}$ and $\phi(\lambda)=\lambda^{r}$, and hence $\phi$ is standard. This shows that every automorphism of $\pi_{1}(M)$ which is induced by an orientation preserving homeomorphism of $M$ is standard, as already was stated in Corollary 2.7. Also from the above computation, we find that (since $a^{2}=r= \pm 1$ ) in this case

$$
g=\left(\begin{array}{ll}
1 & b \\
0 & 1
\end{array}\right) \quad \text { or } \quad\left(\begin{array}{cc}
i & b \\
0 & -i
\end{array}\right)
$$

where $b$ is some complex number.

Now we consider the case where $\phi \in \operatorname{Aut}\left(\pi_{1}(M)\right)$ is induced by an orientation reversing homeomorphism. Then assume $\phi(\mu)=\mu^{r} \lambda^{n}$, where $r=1$ or $-1, n$ is a (possibly zero) integer, and $\phi(\lambda)=\lambda^{-r}$. Again by the above paragraphs, there exists some $g \in \operatorname{PSL}(2, C)$ such that $\phi(U)=\left(A^{*} g\right) U\left(A^{*} g\right)^{-1}$ for every $U \in \pi_{1}(M)$. Since $\left(A^{*}\right)^{-1}=A^{*}$, so if $g=\left(\begin{array}{ll}a & b \\ c & d\end{array}\right)$, then we have

$$
\begin{aligned}
\phi(\mu) & =\phi\left(\left(\begin{array}{ll}
1 & 1 \\
0 & 1
\end{array}\right)\right)=\left(A^{*}\left(\begin{array}{ll}
a & b \\
c & d
\end{array}\right)\right)\left(\begin{array}{ll}
1 & 1 \\
0 & 1
\end{array}\right)\left(A^{*}\left(\begin{array}{ll}
a & b \\
c & d
\end{array}\right)\right)^{-1} \\
& =\left(\begin{array}{cc}
1-a c & a^{2} \\
-c^{2} & 1+a c
\end{array}\right)
\end{aligned}
$$

and

$$
\phi(\lambda)=\phi\left(\left(\begin{array}{ll}
1 & x \\
0 & 1
\end{array}\right)\right)=\overline{\left(\begin{array}{cc}
1-a c x & a^{2} x \\
-c^{2} x & 1+a c x
\end{array}\right)} .
$$

On the other hand, since $\phi(\mu)=\mu^{r} \lambda^{n}$ and $\phi(\lambda)=\lambda^{-r}$, we have

$$
\overline{\left(\begin{array}{cc}
1-a c & a^{2} \\
-c^{2} & 1+a c
\end{array}\right)}=\left(\begin{array}{cc}
1 & r+n x \\
0 & 1
\end{array}\right)
$$

and

$$
\overline{\left(\begin{array}{cc}
1-a c x & a^{2} x \\
-c^{2} x & 1+a c x
\end{array}\right)}=\left(\begin{array}{cc}
1 & -r x \\
0 & 1
\end{array}\right) .
$$

Note that the equalities of matrices in (3) and (4) are in $\operatorname{PSL}(2, C)$.

From (3), we obtain $-\bar{c}^{2}=0$, so $c=0$. From (4), we obtain $\bar{a}^{2} \bar{x}=-r x$; hence $\bar{a}^{2}=-r x / \bar{x}$. By a simple computation, we obtain $\bar{a}= \pm i(x / \bar{x})^{1 / 2}$ according as 
$r=1$ or $r=-1$. Therefore, after simplification, we may write $\phi(\mu)$ in terms of matrices as

$$
\phi(\mu)=\left(\begin{array}{cc}
1 & -r x / \bar{x} \\
0 & 1
\end{array}\right)
$$

and $\phi$ corresponds to $A^{*} g$ where

$$
g=\left(\begin{array}{cc} 
\pm i(\bar{x} / x)^{1 / 2} & b \\
0 & \mp i(x / \bar{x})^{1 / 2}
\end{array}\right) \quad \text { or } \quad\left(\begin{array}{cc} 
\pm(\bar{x} / x)^{1 / 2} & b \\
0 & \pm(x / \bar{x})^{1 / 2}
\end{array}\right)
$$

for some $b \in C$, according as $r=1$ or $r=-1$.

Now if $n \neq 0$, that is, the automorphism $\phi$ is exotic, then we have

THEOREM 2.9. Let $\phi$ be an exotic automorphism of a hyperbolic knot group and let $\lambda=\left(\begin{array}{ll}1 & x \\ 0 & 1\end{array}\right)$ be the longitude of the knot in $\operatorname{PSL}(2, C)$ for some $x \in C-R$. Then $\phi(\mu)=\mu^{r} \lambda^{n}$, where $r=1$ or -1 , and $n=-2 r \operatorname{Re}(x) /\left(|x|^{2}\right)$. Therefore $n$ is uniquely determined (up to sign) by $x$.

Proof. From (3), we also obtain $\bar{a}^{2}=r+n x$. Since $\bar{a}^{2}=-r x / \bar{x}$ from (4), we have $-r x / \bar{x}=r+n x$, and so $-r x=r \bar{x}+n|x|^{2}$, or $n|x|^{2}=-r(x+\bar{x})$, or $n|x|^{2}=-2 r \operatorname{Re}(x)$. Hence $n=-2 r \operatorname{Re}(x) /\left(|x|^{2}\right)$.

By using Theorem 2.9, we may also find a bound for the complex number $x$ in the representation of the longitude of a hyperbolic knot group, in order to have exotic automorphisms.

COROLLARY 2.10. Let $\left(\begin{array}{ll}1 & x \\ 0 & 1\end{array}\right)$ be the longitude of a hyperbolic knot in $\operatorname{PSL}(2, C)$. If the knot group admits an exotic automorphism, then $|x|<2$.

PROOF. Since by Theorem $2.9, n=-2 r \operatorname{Re}(x) /\left(|x|^{2}\right)$, so $\operatorname{Re}(x)=(-r / 2) n|x|^{2}$. Let $x=(-r / 2) n|x|^{2}+b i$ with $b \neq 0$. Then $|x|^{2}=(1 / 4) n^{2}|x|^{4}+b^{2}$, and so $(1 / 4) n^{2}|x|^{4}<|x|^{2}$; or $(1 / 4) n^{2}|x|^{2}<1$; or $|x|<2 /|n|<2$. Thus we have $|x|<2$.

Moreover, if in Theorem $2.9 x$ is pure imaginary, then we have

COROLlaRY 2.11. Let $\left(\begin{array}{ll}1 & x \\ 0 & 1\end{array}\right)$ be the longitude of a hyperbolic knot in $\operatorname{PSL}(2, C)$. If $x$ is a pure imaginary number, then the knot group admits no exotic automorphisms.

Assume there is $g=\left(\begin{array}{ll}a & b \\ 0 & d\end{array}\right) \in \operatorname{PSL}(2, C)$ such that $\left(A^{*} g\right) \mu\left(A^{*} g\right)^{-1}=\mu^{-1}$ and $\left(A^{*} g\right) \lambda\left(A^{*} g\right)^{-1}=\lambda$. Then we have $\bar{a}^{2}=-1$ and $\bar{a}^{2} \bar{x}=x$, so $-\bar{x}=x$; hence $\operatorname{Re}(x)=0$. Thus $x$ is pure imaginary. By Corollary 2.11 , the knot group admits no exotic automorphisms. Similarly, if $\left(A^{*} g\right) \mu\left(A^{*} g\right)^{-1}=\mu$ and $\left(A^{*} g\right) \lambda\left(A^{*} g\right)^{-1}=$ $\lambda^{-1}$, then we have $\bar{a}^{2}=1$ and $\bar{a}^{2} \bar{x}=-x$; and so $\operatorname{Re}(x)=0$, and $x$ is pure complex. Again by Corollary 2.11 the knot group admits no exotic automorphisms. Therefore we obtain a second proof of Corollary 2.8 .

COROLLARY 2.12. Let $\phi$ be an automorphism of a hyperbolic knot group which is induced by an orientation reversing homeomorphism of the knot manifold, such that $\phi$ corresponds to $A^{*} g$ for some $g=\left(\begin{array}{ll}a & b \\ 0 & d\end{array}\right) \in \operatorname{PSL}(2, C)$. Then $\phi$ is standard if and only if $a^{2}=1$ or -1 .

Proof. Assume $\phi$ is standard. Then we have either $\left(A^{*} g\right) \mu\left(A^{*} g\right)^{-1}=\mu^{-1}$ and $\left(A^{*} g\right) \lambda\left(A^{*} g\right)^{-1}=\lambda$; or $\left(A^{*} g\right) \mu\left(A^{*} g\right)^{-1}=\mu$ and $\left(A^{*} g\right) \lambda\left(A^{*} g\right)^{-1}=\lambda^{-1}$. We have seen from the above paragraph that in either case we have $a^{2}=1$ or $a^{2}=-1$. 
Conversely, assume $a^{2}= \pm 1$. Let $\phi(\mu)=\mu \lambda^{n}$ and $\phi(\lambda)=\lambda^{-1}$. Then we have $\bar{a}^{2}=1+n x$, so $1+n x=1$, or $1+n x=-1$. But if $1+n x=-1$, then $x=-2 / n$ is a real number, which is impossible. Therefore $1+n x=1$, and hence $n=0$. The case $\phi(\mu)=\mu^{-1} \lambda^{n}$ and $\phi(\lambda)=\lambda$ can be done similarly.

REMARK. A very recent result of Culler, Gordon, Luecke and Shalen has several consequences for the conjecture considered in this paper. The statement of this result is as follows. Let $K$ be a knot such that $C^{3}(K)$ is not Seifert fibered. Let $\alpha$, $\beta$ be two simple closed curves on $\operatorname{Bdy}\left(C^{3}(K)\right)$ and denote their intersection number by $\Delta(\alpha, \beta)$. Now if $\alpha$ is a $(p, q)$-curve and $\beta$ is an $(m, n)$-curve on $\operatorname{Bdy}\left(C^{3}(K)\right)$, and suppose both $\pi_{1}((K ; p / q))$ and $\pi_{1}((K ; m / n))$ are cyclic, then $\Delta(\alpha, \beta) \leq 1$. This implies that for any knot $K$, there is at most one value of $n \neq 0$ such that $(K ; 1 / n)=S^{3}$, and that value is either 1 or -1 . As a result, several partial results in this paper can be sharpened.

First, it shows that if $K$ is either hyperbolic or a knot considered in the remark following Corollary 2.8, then the only possible exotic algebraic meridian (up to conjugation and inversion) is $\mu \lambda^{\varepsilon}$, where $\varepsilon$ is either 1 or -1 .

Specializing to the case of a hyperbolic knot, Theorem 2.9 then implies that $2 \operatorname{Re}(x)= \pm|x|^{2}$, which is readily shown to be equivalent to $|x \mp 1|=1$.

Finally, it implies that every automorphism of the group of any cable knot is standard. For, in the course of proving Theorem 2.3 it is shown that if $K=$ $J(p, q ; L)$ has an exotic automorphism, then $L$ has an exotic automorphism $h$ such that $h(\mu)=\mu^{a} \lambda^{m}, h(\lambda)=\lambda^{-a}$, where $m=2 a q / p$. This implies that $|p|=1$ or 2 . But if $|p|=1$, then $|m|=2|q| \geq 4$; and if $|p|=2$, then $|m|=|q| \geq 2$. In any case, $|m|>1$, contradicting the CGLS result.

3. Figure-eight knot. Riley has shown in [7] that the figure-eight knot is a hyperbolic knot. For the rest of this paper we will investigate the figure-eight knot group and the automorphisms of this knot group. In particular, we shall exhibit a normalized representation of the figure-eight knot group into $\operatorname{PSL}(2, C)$, find the particular complex number $x$ for the longitude, and then identify matrices of $\operatorname{PSL}(2, C)$ with generators of the group of outer automorphisms of the figure-eight knot group.

By Magnus' 1931 paper [5], the figure-eight knot group $G$ has the following presentation (Dehn presentation):

$$
G=\left\langle a, b, c, d, e: c d^{-1} a=a b^{-1} c=a d^{-1} e b^{-1}=c b^{-1} e d^{-1}=1\right\rangle
$$

The group of automorphisms is generated by automorphisms $f$ and $g$ with

$$
\begin{aligned}
& f: a \rightarrow c^{-1} \quad \text { and } \quad h: a \rightarrow b e^{-1} \\
& b \rightarrow b^{-1} \quad b \rightarrow c e^{-1} \\
& c \rightarrow a^{-1} \quad c \rightarrow d e^{-1} \\
& d \rightarrow d^{-1} \quad d \rightarrow a e^{-1} \\
& e \rightarrow e^{-1} \quad e \rightarrow e^{-1} \text {. }
\end{aligned}
$$


It is easy to see that $f^{2}=h^{4}=1$. We can find $f h$ and $(f h)^{2}$ as follows:

$$
\begin{aligned}
& f h: a \rightarrow b^{-1} e \quad \text { and } \quad(f h)^{2}: a \rightarrow e^{-1} a e \\
& b \rightarrow a^{-1} e \quad b \rightarrow e^{-1} b e \\
& c \rightarrow d^{-1} e \quad c \rightarrow e^{-1} c e \\
& d \rightarrow c^{-1} e \quad d \rightarrow e^{-1} d e \\
& e \rightarrow e \quad e \rightarrow e \text {. }
\end{aligned}
$$

So $(f h)^{2}$ is an inner automorphism. Actually, Magnus showed that the group of outer automorphisms of $G$ has the presentation

$$
\operatorname{Out}(G)=\left\langle f, h: f^{2}=h^{4}=(f h)^{2}=1\right\rangle=\text { dihedral group of order } 8 .
$$

Using the relations, we may simplify the group $G$ as

$$
\begin{array}{rlrl}
G=\left\langle\mu, \beta: \mu \beta^{-1} \mu^{-1} \beta \mu=\right. & \left.\beta \mu \beta^{-1} \mu^{-1} \beta\right\rangle . \\
f: \mu & \rightarrow \beta^{-1} \quad \text { and } \quad h: \mu & \rightarrow \mu \beta^{-1} \mu^{-1} \\
\beta & \rightarrow \mu^{-1} & & \rightarrow \beta \mu^{-1} \beta^{-1} .
\end{array}
$$

Riley showed [7] that there is a faithful representation of $G$ in $\operatorname{PSL}(2, C)$, so that $G$ is discrete in $\operatorname{PSL}(2, C)$ under this representation, and the generators $\mu$ and $\beta$ can be represented as

$$
\mu=\left(\begin{array}{ll}
1 & 1 \\
0 & 1
\end{array}\right) \quad \text { and } \beta=\left(\begin{array}{cc}
1 & 0 \\
-\omega & 1
\end{array}\right),
$$

where $\omega$ is the cube root of $1, \omega=(-1+\sqrt{3} i) / 2$.

We wish to determine the actions of automorphisms $f$ and $h$ in terms of the generators $\mu$ and $\beta$.

Fix a basepoint, and let $\mu$ denote the meridian of the figure-eight knot. By reading around the knot we find that the longitude $\lambda$ can be presented as $\lambda=$ $\beta \mu^{-1} \beta^{-1} \mu^{2} \beta^{-1} \mu^{-1} \beta$ in $G$. Therefore as an element in $\operatorname{PSL}(2, C), \lambda$ can be represented as

$$
\begin{aligned}
\lambda & =\left(\begin{array}{cc}
1 & 0 \\
-\omega & 1
\end{array}\right)\left(\begin{array}{cc}
1 & -1 \\
0 & 1
\end{array}\right)\left(\begin{array}{ll}
1 & 0 \\
\omega & 1
\end{array}\right)\left(\begin{array}{ll}
1 & 2 \\
0 & 1
\end{array}\right)\left(\begin{array}{cc}
1 & 0 \\
\omega & 1
\end{array}\right)\left(\begin{array}{cc}
1 & -1 \\
0 & 1
\end{array}\right)\left(\begin{array}{cc}
1 & 0 \\
-\omega & 1
\end{array}\right) \\
& =\left(\begin{array}{cc}
-1 & -2 \omega+2 \omega^{2} \\
0 & -1
\end{array}\right) .
\end{aligned}
$$

Since $-2 \omega+2 \omega^{2}=2 \sqrt{3} i$, and since $-A$ is identified with $A$ for every $A$ in $\operatorname{PSL}(2, C)$, therefore $\lambda$ can also be represented as

$$
\lambda=\left(\begin{array}{cc}
1 & 2 \sqrt{3} i \\
0 & 1
\end{array}\right) .
$$

Recall that $f(\mu)=\beta^{-1}$. By using the relation $\mu \beta^{-1} \mu^{-1} \beta \mu=\beta \mu \beta^{-1} \mu^{-1} \beta$, we can write

$$
f(\mu)=\beta^{-1}=\left(\mu^{-1} \beta \mu \beta^{-1}\right) \mu^{-1}\left(\beta \mu^{-1} \beta^{-1} \mu\right)=\left(\mu^{-1} \beta \mu \beta^{-1}\right) \mu^{-1}\left(\mu^{-1} \beta \mu \beta^{-1}\right) .
$$


Also since $\lambda=\beta \mu^{-1} \beta^{-1} \mu \mu \beta^{-1} \mu^{-1} \beta$, we have

$$
\beta^{-1} \mu \beta \mu=\lambda^{-1}\left(\beta \mu^{-1} \beta^{-1} \mu\right) \text {. }
$$

Recall also that $f(\beta)=\mu^{-1}$. Hence we have

$$
\begin{aligned}
f(\lambda) & =f\left(\beta \mu^{-1} \beta^{-1} \mu \mu \beta^{-1} \mu^{-1} \beta\right)=\left(\mu^{-1} \beta \mu \beta^{-1}\right)\left(\beta^{-1} \mu \beta \mu^{-1}\right) \\
& =\left(\mu^{-1} \beta \mu \beta^{-1}\right) \lambda^{-1}\left(\beta \mu^{-1} \beta^{-1} \mu\right)=\left(\mu^{-1} \beta \mu \beta^{-1}\right) \lambda^{-1}\left(\mu^{-1} \beta \mu \beta^{-1}\right)^{-1} .
\end{aligned}
$$

So we see that, by letting $y=\mu^{-1} \beta \mu \beta^{-1}$, we have $f(\mu)=y \mu^{-1} y^{-1}$, and $f(\lambda)=$ $y \mu^{-1} y^{-1}$. Let $I_{y}$ denote the inner automorphism of conjugation by $y$, and let $F=I_{y} f$. Then we have $F(\mu)=\mu^{-1}$ and $F(\lambda)=\lambda^{-1}$.

Recall that $h(\mu)=\mu \beta^{-1} \mu^{-1}$. Use the relation $\mu \beta^{-1} \mu^{-1} \beta \mu=\beta \mu \beta^{-1} \mu^{-1} \beta$ to obtain

$$
\beta^{-1}=\mu^{-1} \beta \mu \beta^{-1} \mu^{-1} \beta \mu^{-1} \beta^{-1} \mu .
$$

Therefore

$$
h(\mu)=\mu \beta^{-1} \mu^{-1}=\mu \mu^{-1} \beta \mu \beta^{-1} \mu^{-1} \beta \mu^{-1} \beta^{-1} \mu \mu^{-1}=\left(\beta \mu \beta^{-1}\right)\left(\mu^{-1}\right)\left(\beta \mu^{-1} \beta^{-1}\right) .
$$

Use the relation $\mu \beta^{-1} \mu^{-1} \beta \mu=\beta \mu \beta^{-1} \mu^{-1} \beta$ again to obtain the relations

$$
\mu=\beta \mu^{-1} \beta^{-1} \mu \beta \mu^{-1} \beta \mu \beta^{-1} \text { and } \mu^{-1} \beta=\beta^{-1} \mu^{-1} \beta \mu \beta^{-1} \mu \beta \mu^{-1} \text {. }
$$

Also since $\lambda=\beta \mu^{-1} \beta^{-1} \mu^{2} \beta^{-1} \mu^{-1} \beta$, then $\mu^{2} \beta^{-1} \mu^{-1} \beta=\left(\beta \mu \beta^{-1}\right) \lambda$. We have

$$
\begin{aligned}
h(\lambda)= & h\left(\beta \mu^{-1} \beta^{-1} \mu \mu \beta^{-1} \mu^{-1} \beta\right) \\
= & \beta \mu^{-1} \beta^{-1} \mu \beta \mu^{-1} \beta \mu \beta^{-1} \mu \beta^{-1} \mu^{-1} \mu \beta^{-1} \mu^{-1} \beta \mu \beta^{-1} \mu \beta \mu^{-1} \beta \\
& \cdot\left(\beta \mu^{-1} \beta^{-1} \mu \beta \mu^{-1} \beta \mu \beta^{-1}\right) \mu \beta^{-1} \\
& \cdot\left(\beta^{-1} \mu^{-1} \beta \mu \beta^{-1} \mu \beta \mu^{-1}\right) \beta \mu^{-1} \beta \mu^{2} \beta^{-1}\left(\beta^{-1} \mu^{-1} \beta \mu \beta^{-1} \mu \beta \mu^{-1}\right) \beta \mu^{-1} \beta^{-1} \\
= & \left(\mu^{2} \beta^{-1} \mu^{-1} \beta\right) \beta \mu^{-1} \beta^{-1}\left(\beta \mu \beta^{-1}\right) \lambda\left(\beta \mu^{-1} \beta^{-1}\right)=\left(\beta \mu \beta^{-1}\right) \lambda\left(\beta \mu \beta^{-1}\right)^{-1} .
\end{aligned}
$$

So if we let $z=\beta \mu \beta^{-1}$, then we have $h(\mu)=z \mu^{-1} z^{-1}$, and $h(\lambda)=z \lambda z^{-1}$. Let $I_{z}$ denote the inner automorphism of conjugation by $z$, and let $H=I_{z} h$. Then we have $H(\mu)=\mu^{-1}$ and $H(\lambda)=\lambda$.

We note that $F=f$ and $H=h$ are generators of $\operatorname{Out}(G)$.

We also note that from the actions of $F$ and $H$ on $\mu$ and $\lambda$, we see that the automorphism $F$ is induced by an orientation preserving homeomorphism which reverses both meridian and longitude. This shows the well-known fact that the figure-eight knot is invertible. Also since the automorphism $H$ is induced by a homeomorphism which reverses the meridian but fixes the longitude, this shows another well-known fact that the figure-eight knot is amphicheiral.

Next we shall find matrices representing $F$ and $H$.

Since $F$ is induced by an orientation preserving homeomorphism, there is $g \in \Omega$ the normalizer of $G$ in $\operatorname{P\Gamma L}(2, C)$, and $g \in \operatorname{PSL}(2, C)$ such that $F(x)=g x g^{-1}$ for all $x$ in $G$. In particular, we have $F(\mu)=g \mu g^{-1}, F(\lambda)=g \lambda g^{-1}$ and $F(\beta)=g \beta g^{-1}$. From above we obtain $\mu^{-1}=g \mu g^{-1}, \lambda^{-1}=g \lambda^{-1} g^{-1}$; and since $F(\beta)=\left(I_{y} f\right)(\beta)=$ $I_{y}\left(\mu^{-1}\right)=y \mu^{-1} y^{-1}$, we have $y \mu^{-1} y^{-1}=g \beta g^{-1}$.

Let $g=\left(\begin{array}{ll}a & b \\ c & d\end{array}\right)$. Since $y=\left(\mu^{-1} \beta \mu \beta^{-1}\right)^{-1}=\beta \mu^{-1} \beta^{-1} \mu$,

$$
y=\left(\begin{array}{cc}
1 & 0 \\
-\omega & 0
\end{array}\right)\left(\begin{array}{cc}
1 & -1 \\
0 & 1
\end{array}\right)\left(\begin{array}{ll}
1 & 0 \\
\omega & 1
\end{array}\right)\left(\begin{array}{ll}
1 & 1 \\
0 & 1
\end{array}\right)=\left(\begin{array}{cc}
1-\omega & -\omega \\
\omega^{2} & 0
\end{array}\right)
$$


and so we have

$$
y \mu^{-1} y^{-1}=\left(\begin{array}{cc}
\omega^{2} & -1+2 \omega-\omega^{2} \\
\omega & 2-\omega^{2}
\end{array}\right) .
$$

Thus we obtain the following equations of matrices:

$$
\begin{gathered}
\left(\begin{array}{cc}
1 & -1 \\
0 & 1
\end{array}\right)=\left(\begin{array}{ll}
a & b \\
c & d
\end{array}\right)\left(\begin{array}{ll}
1 & 1 \\
0 & 1
\end{array}\right)\left(\begin{array}{cc}
d & -b \\
-c & a
\end{array}\right) \\
\left(\begin{array}{cc}
\omega^{2} & -1+2 \omega-\omega^{2} \\
\omega & 2-\omega^{2}
\end{array}\right)=\left(\begin{array}{ll}
a & b \\
c & d
\end{array}\right)\left(\begin{array}{cc}
1 & 0 \\
-\omega & 1
\end{array}\right)\left(\begin{array}{cc}
d & -b \\
-c & a
\end{array}\right) .
\end{gathered}
$$

From (1), we have

$$
\left(\begin{array}{cc}
1 & -1 \\
0 & 1
\end{array}\right)=\left(\begin{array}{cc}
1-a c & a^{2} \\
-c^{2} & 1+a c
\end{array}\right) .
$$

So $c=0$ and $a^{2}=-1$, so $a=i$ and $d=-i$, or $a=-i$ and $d=i$. Thus we have

$$
g=\left(\begin{array}{cc}
i & b \\
0 & -i
\end{array}\right) \quad \text { or } \quad g=\left(\begin{array}{cc}
-i & b \\
0 & i
\end{array}\right) \text {. }
$$

First assume $g=\left(\begin{array}{cc}i & b \\ 0 & -i\end{array}\right)$. From (2), we have

$$
\left(\begin{array}{cc}
\omega^{2} & -1+2 \omega-\omega^{2} \\
\omega & 2-\omega^{2}
\end{array}\right)=\left(\begin{array}{cc}
1+i \omega b & b^{2} \omega \\
\omega & 1-i \omega b
\end{array}\right) .
$$

So we have $\omega^{2}=1+i \omega b,-1+2 \omega-\omega^{2}=b^{2} \omega$ and $2-\omega^{2}=1-i \omega b$. Actually, the first equation implies the other two, so by $\omega^{2}=1+i \omega b$, we obtain $b=\omega^{2}-1 / i \omega=\sqrt{3}$. Hence $g$ can be expressed explicity as

$$
g=\left(\begin{array}{cc}
i & \sqrt{3} \\
0 & -i
\end{array}\right)
$$
have

If $g=\left(\begin{array}{cc}-i & b \\ 0 & i\end{array}\right)$, a similar calculation as above will show that $b=-\sqrt{3}$, and so we

$$
g=\left(\begin{array}{cc}
-1 & -\sqrt{3} \\
0 & i
\end{array}\right)
$$

Note that the two matrices

$$
\left(\begin{array}{cc}
i & \sqrt{3} \\
0 & -i
\end{array}\right) \text { and }\left(\begin{array}{cc}
-1 & -\sqrt{3} \\
0 & i
\end{array}\right)
$$

represent the same element in $\operatorname{PSL}(2, C)$.

Since $H$ is induced by an orientation reversing homeomorphism, there is an element $A^{*} g \in \Omega, g \in \operatorname{PSL}(2, C)$, such that $H(x)=\left(A^{*} g\right) x\left(A^{*} g\right)^{-1}$ for all $x \in G$. Therefore we have

$$
\mu^{-1}=H(\mu)=\left(A^{*} g\right) \mu\left(A^{*} g\right)^{-1}, \quad \lambda=H(\lambda)=\left(A^{*} g\right) \lambda\left(A^{*} g\right)^{-1},
$$

and

$$
z \beta \mu^{-1} \beta^{-1} z^{-1}=H(\beta)=\left(A^{*} g\right) \beta\left(A^{*} g\right)^{-1}
$$


Since $z=\left(\beta \mu \beta^{-1}\right)^{-1}=\beta \mu^{-1} \beta^{-1}$, we have

$$
z=\left(\begin{array}{cc}
1 & 0 \\
-\omega & 1
\end{array}\right)\left(\begin{array}{cc}
1 & -1 \\
0 & 1
\end{array}\right)\left(\begin{array}{ll}
1 & 0 \\
\omega & 1
\end{array}\right)=\left(\begin{array}{cc}
1-\omega & -1 \\
\omega^{2} & 1+\omega
\end{array}\right) .
$$

Letting $g=\left(\begin{array}{ll}a & b \\ c & d\end{array}\right)$; we obtain the following equations of matrices:

$$
\left(\begin{array}{cc}
1 & -1 \\
0 & 1
\end{array}\right)=\overline{\left(\begin{array}{ll}
a & b \\
c & d
\end{array}\right)\left(\begin{array}{ll}
1 & 1 \\
0 & 1
\end{array}\right)\left(\begin{array}{cc}
d & -b \\
-c & a
\end{array}\right)}
$$

$$
\begin{gathered}
\left(\begin{array}{cc}
1-\omega & -1 \\
\omega^{2} & 1+\omega
\end{array}\right)\left(\begin{array}{cc}
1 & 0 \\
-\omega & 1
\end{array}\right)\left(\begin{array}{cc}
1 & -1 \\
0 & 1
\end{array}\right)\left(\begin{array}{ll}
1 & 0 \\
\omega & 1
\end{array}\right)\left(\begin{array}{cc}
1+\omega & 1 \\
-\omega^{2} & 1+\omega
\end{array}\right) \\
=\left(\begin{array}{ll}
a & b \\
c & d
\end{array}\right)\left(\begin{array}{cc}
1 & 0 \\
-\omega & 1
\end{array}\right)\left(\begin{array}{cc}
d & -b \\
-c & a
\end{array}\right) .
\end{gathered}
$$

From (3), we obtain

$$
\left(\begin{array}{cc}
1 & -1 \\
0 & 1
\end{array}\right)=\overline{\left(\begin{array}{cc}
1-a c & a^{2} \\
-c^{2} & 1+a c
\end{array}\right)}
$$

So $c=0$ and $a^{2}=-1$, so $a=i$ and $d=-i$, or $a=-i$ and $d=i$. Hence

$$
g=\left(\begin{array}{cc}
i & b \\
0 & -i
\end{array}\right) \quad \text { or } \quad g=\left(\begin{array}{cc}
-i & b \\
0 & i
\end{array}\right)
$$

Let us first assume that $g=\left(\begin{array}{cc}i & b \\ 0 & -i\end{array}\right)$. The left-hand side of $(4)$ is

$$
\begin{aligned}
& \left(\begin{array}{cc}
1-\omega & -1 \\
\omega^{2} & 1+\omega
\end{array}\right)\left(\begin{array}{cc}
1 & 0 \\
-\omega & 1
\end{array}\right)\left(\begin{array}{cc}
1 & -1 \\
0 & 1
\end{array}\right)\left(\begin{array}{cc}
1 & 0 \\
\omega & 1
\end{array}\right)\left(\begin{array}{cc}
1+\omega & 1 \\
-\omega^{2} & 1-\omega
\end{array}\right) \\
& =\left(\begin{array}{cc}
1-\omega & -1 \\
\omega^{2} & 1+\omega
\end{array}\right) .
\end{aligned}
$$

So (4) is equivalent to

$$
\left(\begin{array}{cc}
1-\omega & -1 \\
\omega^{2} & 1+\omega
\end{array}\right)=\overline{\left(\begin{array}{cc}
1+i b \omega & b^{2} \omega \\
\omega & 1-i b \omega
\end{array}\right)}
$$

and we obtain $1-\omega=\overline{1+i b \omega},-1=\bar{b}^{2} \bar{\omega}$, and $1+w=\overline{1-i b w}$. By $-1=\bar{b}^{2} \bar{\omega}$, we have $b^{2} \omega=-1$, or $b^{2}=-1 / \omega=-\omega^{2}$; so $b=i \omega$ or $b=-i \omega$. It is easy to see that $b=-i \omega$ does not satisfy the other two equations; hence $b=i \omega$, and we have $g=\left(\begin{array}{ll}1 & i \omega \\ 0 & -i\end{array}\right)$. Similarly if $g=\left(\begin{array}{cc}-1 & b \\ 0 & i\end{array}\right)$, we can show that $b=-i \omega$, and so $g=\left(\begin{array}{cc}-i & -i \omega \\ 0 & i\end{array}\right)$. These two matrices are the same in $\operatorname{PSL}(2, C)$.

In conclusion, the group of outer automorphisms of the figure-eight knot group is the dihedral group of order eight, with two generators $f$ and $h$, where $f$ corresponds to the matrix

$$
\left(\begin{array}{cc}
i & \sqrt{3} \\
0 & -i
\end{array}\right)
$$


and $h$ corresponds to

$$
A^{*}\left(\begin{array}{cc}
i & i \omega \\
0 & -i
\end{array}\right)
$$

where $\omega=(-1+\sqrt{3} i) / 2$ is a cube root of 1 . Also we can represent the longitude $\lambda$ as

$$
\lambda=\left(\begin{array}{cc}
1 & 2 \sqrt{3} i \\
0 & 1
\end{array}\right) .
$$

Notice that by any one of Corollaries 2.8, 2.10, 2.11, and 2.12, the figure-eight knot group admits no exotic automorphisms. It is also interesting to note that each of the matrices

$$
\left(\begin{array}{cc}
i & \sqrt{3} \\
0 & -i
\end{array}\right) \text { and }\left(\begin{array}{cc}
i & -\omega \\
0 & i
\end{array}\right)
$$

has order 2 , and

$$
\left(A^{*}\left(\begin{array}{cc}
i & i \omega \\
0 & -i
\end{array}\right)\right)^{4}=\lambda
$$

\section{REFERENCES}

1. E. M. Brown, Unknotting in $M^{2} \times I$, Trans. Amer. Math. Soc. 123 (1966), 480-505.

2. C. Feustel and W. Whitten, Groups and complements of knots, Canad. J. Math. 30 (1978), 1284-1295.

3. K. Johannson, Homotopy equivalences of 3-manifolds with boundaries, Lecture Notes in Math., Vol. 761, Springer-Verlag, Berlin and New York, 1979.

4. R. Litherland, Surgery on knots in solid tori. II, J. London Math. Soc. 22 (1980), 559-569.

5. W. Magnus, Untersuchungen über einige unendliche diskontinuierliche Gruppen, Math. Ann. 105 (1931), 52-74.

6. A. Marsden, The geometry of finitely generated Kleinian groups, Ann. of Math. (2) 99 (1974), 383-462.

7. R. Riley, A quadratic parabolic group, Math. Proc. Cambridge Philos. Soc. 77 (1975), 281-288.

8.

9. H. Schubert, Knoten und Vollringe, Acta Math. 90 (1953), 131-286.

10. O. Schreier, Über die Gruppen $A^{a} B^{b}=1$, Abh. Math. Sem. Univ. Hamburg 3 (1923), 167-169.

11. J. Simon, Some classes of knots with Property P, Topology of Manifolds, Markham, Chicago, Ill., 1970.

12. W. Thurston, The geometry and topology of 3-manifolds, Lecture notes, Princeton Univ.

13. F. Waldhausen, On irreducible 3-manifolds which are sufficiently large, Ann. of Math. (2) 87 (1968), 56-88.

14. , Gruppen mit Zentrum und 3-dimensionale Mannigfaltigkeiten, Topology 6 (1967), 505-517.

Department of MAthematics, St. Louis University, St. Louis, Missouri 63103 